\title{
Subtype-Specific Associations Between leukocyte mtDNA Copy Number and the Survival of Early-Sage Breast Cancer
}

\author{
Wenzhe Zhang \\ Fujian Medical University Union Hospital \\ Songping Lin \\ Quanzhou First Hospital Affiliated to Fujian Medical University \\ Bangwei Zeng \\ Fujian Medical University Union Hospital \\ Xiaobin Chen \\ Fujian Medical University Union Hospital \\ Lili Chen \\ Fujian Medical University Union Hospital \\ Minyan Chen \\ Fujian Medical University Union Hospital \\ Wenhui Guo \\ Fujian Medical University Union Hospital \\ Yuxiang Lin \\ Fujian Medical University Union Hospital \\ Liuwen Yu \\ Fujian Medical University Union Hospital \\ Jialin Hou \\ Fujian Medical University Union Hospital \\ Yan Li \\ Fujian Medical University Union Hospital \\ Shengmei Li \\ Fujian Medical University Union Hospital \\ Xuan Jin \\ Fujian Medical University Union Hospital \\ Weifeng Cai \\ Fujian Medical University Union Hospital \\ Kun Zhang \\ Fujian Medical University Union Hospital \\ Qian Nie \\ Fujian Medical University Union Hospital \\ Hanxi Chen \\ Fujian Medical University Union Hospital \\ Jing Li \\ Fujian Medical University Union Hospital \\ Peng He \\ Fujian Medical University Union Hospital \\ Qindong Cai \\ Fujian Medical University Union Hospital \\ Yibin Qiu \\ Fujian Medical University Union Hospital \\ Chuan Wang \\ Fujian Medical University Union Hospital \\ Fangmeng Fu ( $\nabla$ ffm@fjmu.edu.cn ) \\ Fujian Medical University Union Hospital
}

\section{Research Article}

Keywords: Breast cancer, Mitochondrial DNA copy number, Leukocyte, Survival 
Posted Date: February 9th, 2022

DOI: https://doi.org/10.21203/rs.3.rs-1223162/v1

License: @ (i) This work is licensed under a Creative Commons Attribution 4.0 International License. Read Full License 


\section{Abstract}

Background: Compelling evidence has indicated a significant association between leukocyte mitochondrial DNA copy number (mtDNAcn) and prognosis of several malignancies in a cancer-specific manner. However, whether leukocyte mtDNAcn can predict the clinical outcome of breast cancer (BC) patients has not been well investigated.

Methods: The mtDNA copy number of peripheral blood leukocytes from $661 \mathrm{BC}$ patients was measured using a Multiplex AccuCopy ${ }^{\mathrm{T}} \mathrm{Kit}$ based on a multiplex fluorescence competitive PCR principle. Kaplan-Meier curves and Cox proportional hazards regression model were applied to investigate the association of mtDNAcn with invasive disease-free survival (iDFS), distant disease-free survival (DDFS), breast cancer special survival (BCSS), and overall survival (OS) of patients. The possible mtDNAcn-environment interactions were also evaluated by the Cox proportional hazard regression models.

Results: Breast cancer (BC) patients with higher leukocyte mtDNA-CN exhibited a significantly worse iDFS than those with lower leukocyte mtDNAcn (5-year iDFS: fully-adjusted model: $\mathrm{HR}=1.433[95 \% \mathrm{Cl} 1.038-1.978], P=0.028$ ). Interaction analyses showed that mtDNAcn was significantly associated with hormone receptor status (adjusted $\mathrm{p}$ for interaction: 5-year BCSS: 0.028, 5-year OS: 0.022), so that further analysis was mainly in the HR subgroup. Multivariate Cox regression analysis demonstrated that mtDNAcn was an independent prognostic factor for both BCSS and OS in HR-positive patients (HR+: 5-year BCSS: adjusted $\mathrm{HR}(\mathrm{aHR})=2.340[95 \% \mathrm{Cl} 1.163-4.708], P=0.017$ and 5-year OS: aHR = 2.446 [95\% Cl 1.218-4.913], $P=0.011)$.

Conclusions: For the first time, our study demonstrated that leukocyte mtDNA copy number might influence the outcome of early-stage breast cancer patients depending on intrinsic tumor subtypes in Chinese women.

\section{Introduction}

Breast cancer (BC) is the first common malignancy and the fifth leading cause of cancer death among women in China. BC is highly heterogeneous at molecular and clinical levels [1]. Generally, some prognostic factors for early-stage breast cancer (EBC) survival include tumor size, lymph node involvement, tumor grade, and hormone receptor (HR) status. The survival rate has increased due to improved management strategies in the past few decades. However, EBC patients with the same traditional stage often show different clinical outcomes because of tumor heterogeneity.

Consequently, biomarkers are urgently needed to complement the traditional system for more accurate prognostic prediction of EBC and thus benefit individualized treatment. Some theories indicate that genetic background is an important determinant of metastatic potential. The theories also suggest that it might be possible to define metastasis susceptibility based on gene variation in readily accessible tissues (for example, blood) rather than tumors [2]. The study of mtDNA copy number variation in breast cancer is of particular interest, given the role of oxidative stress in the disease's etiology.

Mitochondria are essential cellular organelles in eukaryotic cells and perform multiple cellular functions, notably in energy metabolism, reactive oxygen species (ROS) generation, and apoptosis [3]. Mitochondria contain their DNA (mtDNA), which is susceptible to damage caused by high levels of reactive oxygen species owing to the lack of protective histones and its diminished DNA repair capacity [4]. There can be several 100 or even more than 1000 copies of mitochondrial genomes in each eukaryotic cell. Although mtDNA copy number is significantly varied among cells from different tissue origins [5], it has been observed that there is a good correlation of the number of mtDNA in various cell types within the same individual [6]. The mtDNA copy number may change significantly under different internal or external microenvironments, damaging the OXPHOS system and the enhanced ROS production. This scenario has been believed to contribute to the initiation and development of tumors [7].

Quantitative changes in mtDNA content have been observed in a variety of malignant tumors, such as breast cancer [8], gastric cancer [9], head and neck cancer [10], and colorectal cancer [11]. Previous studies have also shown that changes in mtDNA content in tumor tissues were related to tumor staging, prognosis, and treatment response, again in a cancer type-specific manner [12, 13]. Yu et al. have found that reduced mitochondrial DNA copy number in tumor tissues was correlated with tumor progression and prognosis in Chinese breast cancer patients [8]. There has been a series of reports on the relationship between mtDNA content in peripheral blood leukocytes and cancer susceptibility in recent years [14-17]. However, only a few studies have shown that higher mtDNA content in peripheral blood leukocytes is associated with poor prognosis in colorectal cancer [18], hepatocellular carcinoma [19], and glioma [20]. In addition, Xia et al. have found that leukocyte mtDNA content is associated with the T stage of breast cancer, indicating that high mtDNA content may facilitate cancer progression, but did not explore the relationship with the prognosis of breast cancer [21]. Thus, the effect of mtDNA copy number in peripheral blood leukocytes on BC patient prognosis has not been explored.

This study measured the leukocyte mtDNA copy number in peripheral blood from EBC patients and analyzed the association between leukocyte mtDNA copy number and the survival of patients in Southeastern China with EBC. To the best of our knowledge, this is the first study to assess mtDNA content's value in predicting the prognosis of EBC patients.

\section{Materials And Methods}

\section{Study population}

Our hospital-based study recruited 681 breast cancer cases from Fujian Medical University Union Hospital from July 2000 and October 2014 . Patient eligibility criteria in this study were as follows: (i) histopathologically confirmed with invasive breast cancer; (ii) subsequently treated with curative surgical resection and systemic therapy; (iii) availability of complete clinical and follow-up data $($ (iv) no history of other malignancy and (v) alive at least 1 month after surgery. We ruled out 20 patients, containing 6 patients with breast carcinoma in situ and 14 patients with incomplete clinical information according to this screening criteria. Finally, 661 patients with resected breast cancer were included in the present study for predictive analysis. 
Clinicopathological and demographic information was collected from the hospital records, and survival data were obtained from the followed-up database renewed annually. The patients were staged according to the $7^{\text {th }}$ version of the American Joint Commission on Cancer (AJCC) tumor-node-metastasis (TNM) staging system [22]. Estrogen receptor (ER)/progesterone receptor (PR) positivity was determined by IHC analysis of the number of positively stained nuclei ( $\geq$ $10 \%$ ), and hormone receptor (HR) positivity was defined as being either ER+ and/or PR+. Tumors were considered human epidermal growth factor-2 (HER2) positive when cells exhibited strong membrane staining (3+). Expressions of 2+ would require further in situ hybridization testing for HER2 gene amplification, while expressions of 0 or $1+$ were regarded as negative. The subtypes were categorized [23]: Iuminal A (ER+, PR+ \20\%, HER2-, Ki67囚14\% or grade I when Ki67 was unavailable), luminal B (HR+, HER2-, Ki67 $14 \%$ or grade II/III when Ki67 was unavailable or HR+, HER2+); HER2 enriched (HR-, HER2+) and triple-negative (HR- and HER2-). The Institutional Ethics Committee approved the study, and all participants consented to the testing at the time of their participation and contributed data.

\section{DNA extraction}

Blood samples were collected in EDTA anticoagulant tubes and were centrifuged within 30 min. Genomic DNA was extracted from blood samples by using the Whole-Blood DNA Extraction Kit (Bioteke, Beijing, China), according to the manufacturer's protocol, and then the genomic DNA was aliquoted and stored at $-80^{\circ} \mathrm{C}$ for future analysis. The purity of the DNA samples was assessed before mtDNA copy number assessment, and samples without sufficient DNA yield were not included for mtDNA copy number analysis.

\section{MtDNA copy number assessment}

Relative mtDNA content was measured by a custom-by-design Multiplex AccuCopy ${ }^{\text {TM}}$ Kit (Genesky Biotechnologies Inc., Shanghai, China) based on a multiplex fluorescence competitive PCR principle as previously described [24], which can interrogate copy number variation (CNV) status at multiple genomic loci in the same assay reaction.

The methods below briefly describe the manufacturer's process. A total of 2 target genomic segments within the ND1 gene and 6 reference segments (2, 10, $16,18,19$, and 20 p) were chosen for the AccuCopy assay. The primers for both target and reference segments were synthesized. The forward primers were fluorescent-labeled at Genesky Biotechnologies (Shanghai, China). The competitive DNAs for the ND1 and 6 reference segments were synthesized in doublestrand and provided a mixture from Genesky Biotechnologies (Shanghai, China). These competitive DNAs are almost identical to their homologies in the human reference genome except for 1-2 base pairs deleted. The primers of target segments and reference segments and probes information were provided in Table S1.

The PCR reaction was prepared in $20 \mu \mathrm{l}$ for each sample, containing a mixture of $2 \mu \mathrm{l}$ target genomic DNA (5 ng/ $\mu$ l) with $2 \mu \mathrm{l}$ reference segment DNA, $1 \mu \mathrm{l}$ Multiplex PCR Fluorescence Primer Mix (AccuCopy ${ }^{T M}$ ), $10 \mu \mathrm{l} 2 \times$ PCR Master Mix (Genesky Biotechnologies), and $5 \mu \mathrm{lddH} 2 \mathrm{O} .10 \mu \mathrm{l} 2 \times \mathrm{PCR}$ Master Mix (Genesky Biotechnologies), and $5 \mu \mathrm{lddH} 2 \mathrm{O}$. The program used was an initial denaturation step of $95^{\circ} \mathrm{C}$ for 10 min followed by the first 11 cycles of denaturation at $94^{\circ} \mathrm{C}$ for $20 \mathrm{~s}$, annealing at $60^{\circ} \mathrm{C}$ for $40 \mathrm{~s}$ (the annealing temperature was decreased by $0.5^{\circ} \mathrm{C}$ in each consecutive cycle), and elongation at $72^{\circ} \mathrm{C}$ for $1.5 \mathrm{~min}$, followed by the second 24 cycles of denaturation at $94^{\circ} \mathrm{C}$ for $20 \mathrm{~s}$, annealing at $59^{\circ} \mathrm{C}$ for $30 \mathrm{~s}$, and elongation at $72^{\circ} \mathrm{C}$ for $1.5 \mathrm{~min}$, and a final extension step at $60^{\circ} \mathrm{C}$ for $60 \mathrm{~min}$ and then at $4{ }^{\circ} \mathrm{C}$ forever. The PCR product was diluted in a 1:5 ratio, and $1 \mu$ l diluted products were mixed with $0.5 \mu \mathrm{l} 500$ ( Liz) size standard and $8.5 \mu \mathrm{l} \mathrm{Hi-Di} \mathrm{formamide} \mathrm{(both} \mathrm{from} \mathrm{Applied} \mathrm{Biosystems,} \mathrm{Foster} \mathrm{City,} \mathrm{CA).} \mathrm{The} \mathrm{mixture} \mathrm{was} \mathrm{subjected} \mathrm{to} \mathrm{a} \mathrm{denaturation} \mathrm{step} \mathrm{of} 95^{\circ} \mathrm{C}$ for 5 min and electrophoresed in a $3730 \mathrm{XL}$ genetic analyzer ( $\mathrm{ABI}$, Carlsbad, $\mathrm{CA}, \mathrm{USA}$ ). Raw data were analyzed by GeneMapper 4.0 (ABI). The height and area data for all specific peaks were exported into a Microsoft Excel file.

The sample/competitive (S/C) peak ratio was calculated for the 2 target and 6 reference segments. The S/C ratio for each target fragment was first normalized based on four reference segments, respectively. The 6 normalized S/C ratios were further normalized to the median value in all samples for each reference segment, respectively, and then averaged. If one of the six normalized S/C ratios deviated $₫ 25 \%$ from the average of the other five, it was excluded from further analysis. The copy number of each target segment was determined by the average S/C ratio times two, given that the copy numbers of four reference segments are two in the diploid genome. Twenty samples were randomly selected as blinded duplicates for quality assessment purposes, and excellent concordance was obtained.

\section{Statistical analyses}

Overall survival (OS) and breast cancer-specific survival (BCSS) were our primary endpoints and defined as the time from cancer diagnosis to the date of mortality for all causes and breast cancer, respectively. Invasive disease-free survival (iDFS) and distant disease-free survival (DDFS) were our secondary endpoints and calculated separately from the date of diagnosis to the date of any recurrence and distant recurrence to follow-up cut-off time [25]. Survival data were analyzed using the Kaplan-Meier method with the log-rank test. The latest follow-up date was 2016.12.31, and the median follow-up duration was 78.8 months (ranging from 4 to 189 months). To adjust for possible confounders and evaluate the form of the relationship, Cox proportional hazards models were fitted using different adjustment parameters: model 1 adjusted for age at diagnosis; model 2 additionally adjusted for hormone receptor status, and HER2 status; model 3 was the same as model 2 plus an adjustment for tumor size, Lymph node involvement, and grade. The Cox proportional hazards model results showed the non-linearity in the effects of mitochondrial copy number on cancer progression. Moreover, nonlinear $p$-splines were created to evalutate the functional form of the relationship between mtDNA copy number and BC patients' survival. The results of the splines and the estimates for the quintiles 2 , 3,4 and 5 compared with quintile 1 revealed that a categorical analysis of the data by combining the 2nd, 3rd, 4th and 5th quintile of mtDNA copy number and to set quintile 1 as a reference fits the data better than modelling the data on a continuous scale. The Chi-square test was used to examine differences of categorical variables between subgroups. The student's t-test was used to analyze the difference of normally distributed continuous variables between two groups. The Cox regression model calculated the hazard ratios (HRs) and 95\% confidence interval (Cl) for each factor in multivariate analyses. The possible 
mtDNAcn-environment interactions were also evaluated by the Cox proportional hazard regression models. All tests were 2 -sided, and $P$-values $<0.05$ were considered statistically significant. SAS 9.4 (SAS Institute Inc., Cary, NC) was used for all statistical analyses.

\section{Results}

\section{Patient characteristics and clinical features}

Participants in the 661 early breast cancer cohort were female, and their mean age was $46.73 \pm 10.28$ years old at breast cancer diagnosis. The clinical characteristics and survival of the $661 \mathrm{BC}$ patients are summarized in Table 1. During a follow-up time of 5 years, 279 cases experienced recurrence (61 locoregional and 254 distant), and 180 died (176 died of BC and 4 died of another disease). No significant difference in BC-iDFS, BC-DDFS, BCSS, and OS was shown in the subgroup of age at diagnosis $40(P=0.136,0.331,0.754$ and 0.934$)$. Nevertheless, patients with a tumor size $>2 \mathrm{~cm}$, lymph node-positive, grade III, clinical-stage II + III, or HER2 positive had significantly shorter survival times, whereas HR positivity remarkably improved the survival of BC patients (logrank $P<0.05$, Table 1). Furthermore, our intrinsic molecular subtypes (luminal A, luminal B, HER2-enriched, and triple-negative) were also associated with significantly different survival (log-rank $P<0.05$, Table 1 ).

\section{Effects of mtDNA copy number on survival of BC}

We performed a Cox regression analysis based on quintile categorization of mtDNA copy number applying different adjustments for confounders. Since the estimates for quintiles $2,3,4$, and 5 pointed in the same direction with no clear indication of a linear association, we set the first quintile as the reference category to illustrate the quintiles 2-5 values' influence. Details for each quintile and adjustment model can be found in Supplementary Table S2. In line with this observation, nonlinear splines showed increased risk for patients within the 2-5 quintiles of mtDNA copies, compared with quintile 1 for all 4 outcome variables (Figure S1). For reasons of statistical power, we collapsed quintiles 2-5 and compared it to quintile 1, which was a reference in all subsequent analyses. The detailed characteristics comparing combined quintiles 2-5 against quintile 1 showed in Table 2. Patients with higher copy numbers (quintiles $2-$ 5) have higher grades $(P=0.041)$. The Kaplan-Meier curves and log-rank tests comparing combined quintiles 2-5 against the quintile 1 (Figure 1$)$ showed significant differences concerning 5-year iDFS, DDFS, and OS, except for BCSS ( $P=0.018$ for iDFS, $P=0.042$ for DDFS, $P=0.061$ for BCSS, and $P=0.043$ for OS).

Cox regression analyses model 1 in Table 3 show that higher mtDNA copy number (quintile 2-5) was significantly associated with a 1.476-fold higher risk of 5year $\mathrm{BC}$-specific recurrence ( $\mathrm{HR}=1.476[95 \% \mathrm{Cl} 1.073-2.031], P=0.016), 1.418$-fold higher risk of 5-year distant recurrence $(\mathrm{HR}=1.412[95 \% \mathrm{Cl} 1.015-$ 1.981], $P=0.040)$, and 1.522-fold higher risk of 5 -year overall death ( $\mathrm{HR}=1.522[95 \% \mathrm{Cl} 1.011-2.291], P=0.044)$. However, after adjusting for age at breast cancer diagnosis, tumor size, lymph node involvement, grade, hormone receptor status, and HER2 status, only association with iDFS remained significant. In model 3, quintile 2-5 was significantly associated with shorter iDFS (5-year iDFS: adjusted HR=1.433[95\% Cl 1.038-1.978], $P=0.028$; Table3).

\section{Stratification and interaction analysis}

The associations between mtDNA copy number and BC survival were then evaluated by stratified analysis of age at diagnosis, tumor size, lymph node involvement, grade, hormone-receptor status, and HER2 status, while interaction analysis was also performed between these covariates and mtDNAcn on BC survival. Statistically significant multiplicative interactions on BC survival were found between mtDNA copy number and hormone-receptor status (adjusted $\mathrm{p}$ for interaction: 5-year BCSS: 0.028, 5-year OS: 0.022).

\section{Prognostic analysis in HR+ patients}

As shown in Table 4, we found that higher mtDNA copy numbers (quintile 2-5) were associated with shorter survival of the patients who were positive hormone-receptor status (HR+: 5-year BCSS: aHR $=2.340[95 \% \mathrm{Cl} 1.163-4.708], P=0.017$ and 5-year OS: aHR $=2.446[95 \% \mathrm{Cl} 1.218-4.913], P=0.011$ ). However, compared with HR-positive patients, the performance of mitochondrial copy number in HR-negative patients is the opposite and has no significance (Table4). We further conducted a stepwise Cox proportional hazard analysis to evaluate the effects of clinical variables and mtDNA copy number on BCSS and OS in HR-positive patients. Three variables (node status, grade, mtDNAcn) were selected into the final regression model of breast cancer-specific survival, while four variables (node status, mtDNAcn, grade, HER2) were selected into the final regression model of overall survival (Table 5). In the HR-positive subgroup final model, the mtDNAcn was an independent risk factor for 5-year BCSS with a 2.240-fold ( $95 \% \mathrm{Cl}, 1.204-4.863)$ increased risk and for 5-year OS with a 2.464 -fold $(95 \% \mathrm{Cl}, 1.227-4.948)$ increased risk.

\section{Discussion}

This study evaluated the possible relation between mtDNA copy number in peripheral blood and BC clinical outcome in a cohort of $661 \mathrm{EBC}$ cases. We found that patients with high mtDNA copy numbers showed significantly shorter iDFS than those with low mtDNA (5-year iDFS: adjusted HR=1.433[95\% CI 1.0381.978], $P=0.028$ ). The most significant and novel result of this study is that, in the HR+ subgroup, patients with high copy number have a worse prognosis than those with low mtDNA (HR+: 5-year BCSS: aHR = 2.340[95\% Cl 1.163-4.708], $P=0.017$ and 5-year OS: aHR $=2.446[95 \% \mathrm{Cl} 1.218-4.913], P=0.011$ ). In short, the high mtDNA copy number in peripheral blood led to a worse prognosis for EBC patients, especially in the HR-positive subgroup patients.

Previous studies have proposed that high leukocyte mtDNA copy number in patients may impair the immune defense against tumor cells and lead to poor prognosis. MtDNA content is regulated by a wide range of environmental and genetic factors. For example, raised mtDNA content has been associated with altered oxidative stress, aging, immune response activation, and environmental exposures [26-28]. Several studies have demonstrated that high leukocyte mtDNA content is associated with a worse prognosis of patients with glioma [20], with HCC [19], and with colorectal cancer [18]. These studies have reported that cancer patients with high leukocyte mtDNAcn may be in the immunosuppressive state. Furthermore, mitochondrial ROS, generally increased with mtDNA 
copy number, can inhibit the expansion and functions of helper T cells and killer cells in peripheral blood [29, 30]. In line with these findings, our study demonstrated that a higher leukocyte mtDNA copy number was associated with poor prognosis in HR-positive EBC patients.

However, several reports showed that lower mtDNAcn in breast tumor tissues was associated with a worse prognosis [8, 31, 32]. Only one previous study evaluated the progression role of mtDNA copy number in peripheral blood leucocytes in breast cancer patients. Xia et al. reported that leukocyte mtDNA content is associated with the T stage of breast cancer, indicating that high mtDNA content may facilitate cancer progression [21]. Nevertheless, the relationship between tumor size and prognosis is unstable, and Xia et al. did not have any follow-up data. We are the first and only study to explore the relationship between the mitochondrial DNA copy number of peripheral blood cells and the prognosis of breast cancer patients. We mainly found that higher leukocyte mtDNA content was associated with poor BCSS and OS in HR-positive EBC patients.

In contrast to the observation of mtDNAcn reduction in breast tumor tissues, the studies of leukocyte mtDNAcn of breast cancer patients and its association with prognosis have been heterogeneous. Rai et al. showed an inverse relation in blood vs. tissue samples in breast cancer patients [33]. Our results were consistent with a meta-analysis that summarized the results of 18 studies involving 3961 cancer patients and concluded that elevated mtDNAcns in peripheral blood leukocytes and tumor tissues predict the opposite outcome of cancer [34]. Biologically, mtDNAcn is specific to different tissue types, reflecting differing energy requirements $[35,36]$. Collectively, these findings support the point of view that changes in mtDNA content are involved in the progression of breast cancer.

We observed that high mtDNA content was significantly associated with poor prognosis, mainly in the HR-positive subgroup, but not in the HR-negative subgroup, indicating host characteristics' modulating effects through unknown mechanisms. Hormone receptor (HR) positivity was either ER+ and/or PR+. After binding to estrogen or progesterone, HR can interact with the mtDNA to maintain mitochondrial function, inhibit cell apoptosis, and overcome oxidative damage $[37,38]$. The different performance of mtDNA copy numbers in the HR+ and HR-subgroups may be due to this interaction. Gao et al. found that estrogen indirectly regulated mitochondrial DNA replication and repair functions $[39,40]$. Previous studies have identified that instability of mtDNA due to exposure to estrogen induces cancer cell metastasis [41]. As a result, we proposed that in HR-positive patients, the oxidative damage in the tumor caused by estrogen or progesterone and the immunosuppression of the high copy number in the peripheral blood cells together lead to a significantly worse prognosis.

As the first exploration of a possible relationship between leukocyte mtDNAcn and survival in EBC patients, our study has several strengths, including longterm follow-up, more cases than previous studies, as well as comprehensive clinical data. There are several limitations to our study. First, as all patients were of Chinese origin, it is unclear whether our findings are Chinese Han population-specific or common in other populations. Second, we only performed association analyses between mtDNA content and prognosis of BC patients. The underlying mechanisms through which mtDNA content affects immune functions need further investigation. Lastly, future correlation analysis of mtDNAcn in different tissues (e.g., leukocytes vs. tumor tissues) would help us better understand the relationship between mitochondrial biology and carcinogenesis.

\section{Conclusions}

For the first time, we proposed that the prognostic value of leukocyte mtDNA content in EBC patients may depend on intrinsic tumor subtypes. Our study reminds us that we should pay special attention to HR-positive BC patients with high peripheral blood mitochondrial copy numbers when treating them because the prognosis of HR-positive BC patients may be worse. Once confirmed, leukocyte mtDNA content can serve as a useful biomarker to improve the prognosis prediction for $\mathrm{BC}$ patients.

\section{Abbreviations}

mtDNAcn: mitochondrial DNA copy number; BC: breast cancer; EBC: early-stage breast cancer; iDFS: invasive disease-free survival; DDFS: distant disease-free survival; BCSS: breast cancer special survival; OS: overall survival; HR: hormone receptor; ROS: reactive oxygen species; AJCC: American Joint Commission on Cancer; TNM: tumor-node-metastasis; ER: estrogen receptor; PR: progesterone receptor; HER2: human epidermal growth factor-2; HRs: hazard ratios; Cl: confidence interval; aHR: adjusted hazard ratio.

\section{Declarations}

\section{Ethics approval and consent to participate}

The study protocol was approved by the ethics committee of Fujian Medical University Union Hospital, written informed consent was obtained from all subjects before their participation. All the procedures performed in studies involving human participants adhere to The Code of Ethics of the World Medical Association (Declaration of Helsinki).

\section{Consent for publication}

We have obtained consent to publish from the participants. 
The data that support the findings of this study are available from Fujian Medical University Union Hospital but restrictions apply to the availability of these data, which were used under license for the current study, and so are not publicly available. Data are however available from the authors upon reasonable request and with permission of Fujian Medical University Union Hospital. Fangmeng Fu, the corresponding author, should be contacted if someone wants to request the data from this study.

\section{Competing interests}

The authors declare that they have no conflicts of interest.

Funding

This work was supported by Joint Funds for the Innovation of Science and Technology, Fujian Province [grant numbers 2018Y9055, 2019Y9103, 2020Y9053]; Joint Key Funds for the Health and Education of Fujian Province [grant number 2019-WJ-23]; Joint Key Funds for the Natural Science Foundation of Fujian Province [grant number 2021J01737].

\section{Authors' Contributions}

Chuan Wang and Fangmeng Fu conceived and designed the study. Yan Li, Shengmei Li, Xuan Jin, Weifeng Cai, Kun Zhang, Qian Nie, Hanxi Chen, Jing Li, Peng He, Qindong Cai and Yibin Qiu collected the data. Liuwen Yu and Jialin Hou analyzed the data. Xiaobin Chen, Lili Chen, Minyan Chen, Wenhui Guo, and Yuxiang Lin offer technical assistance. Wenzhe Zhang, Songping Lin, and Bangwei Zeng wrote the manuscript. All authors contributed toward data analysis, drafting, and critically revising the paper, gave final approval of the version to be published, and agreed to be accountable for all aspects of the work.

\section{Acknowledgements}

Not applicable.

\section{References}

1. D'Aiuto F, Callari M, Dugo M, Merlino G, Musella V, Miodini P, Paolini B, Cappelletti V, Daidone MG: miR-30e* is an independent subtype-specific prognostic marker in breast cancer. Br J Cancer 2015, 113(2):290-298.

2. Hunter K, Welch DR, Liu ET: Genetic background is an important determinant of metastatic potential. Nat Genet 2003, 34(1):23-24; author reply 25.

3. Greaves LC, Reeve AK, Taylor RW, Turnbull DM: Mitochondrial DNA and disease. J Pathol 2012, 226(2):274-286.

4. Van Houten B, Woshner V, Santos JH: Role of mitochondrial DNA in toxic responses to oxidative stress. DNA Repair (Amst) 2006, 5(2):145-152.

5. Veltri KL, Espiritu M, Singh G: Distinct genomic copy number in mitochondria of different mammalian organs. J Cell Physio/ 1990, 143(1):160-164.

6. Shen J, Platek M, Mahasneh A, Ambrosone CB, Zhao H: Mitochondrial copy number and risk of breast cancer: a pilot study. Mitochondrion 2010, 10(1):62-68.

7. Lee HC, Wei YH: Mitochondrial DNA instability and metabolic shift in human cancers. Int J Mol Sci 2009, 10(2):674-701.

8. Yu M, Zhou Y, Shi Y, Ning L, Yang Y, Wei X, Zhang N, Hao X, Niu R: Reduced mitochondrial DNA copy number is correlated with tumor progression and prognosis in Chinese breast cancer patients. IUBMB Life 2007, 59(7):450-457.

9. Wu CW, Yin PH, Hung WY, Li AF, Li SH, Chi CW, Wei YH, Lee HC: Mitochondrial DNA mutations and mitochondrial DNA depletion in gastric cancer. Genes Chromosomes Cancer 2005, 44(1):19-28.

10. Kim MM, Clinger JD, Masayesva BG, Ha PK, Zahurak ML, Westra WH, Califano JA: Mitochondrial DNA quantity increases with histopathologic grade in premalignant and malignant head and neck lesions. Clin Cancer Res 2004, 10(24):8512-8515.

11. van Osch FH, Voets AM, Schouten LJ, Gottschalk RW, Simons CC, van Engeland M, Lentjes MH, van den Brandt PA, Smeets HJ, Weijenberg MP: Mitochondrial DNA copy number in colorectal cancer: between tissue comparisons, clinicopathological characteristics and survival. Carcinogenesis 2015 , 36(12):1502-1510.

12. Mizumachi T, Suzuki S, Naito A, Carcel-Trullols J, Evans TT, Spring PM, Oridate N, Furuta Y, Fukuda S, Higuchi M: Increased mitochondrial DNA induces acquired docetaxel resistance in head and neck cancer cells. Oncogene 2008, 27(6):831-838.

13. Hsu CW, Yin PH, Lee HC, Chi CW, Tseng LM: Mitochondrial DNA content as a potential marker to predict response to anthracycline in breast cancer patients. Breast J 2010, 16(3):264-270.

14. Gentiluomo M, Katzke VA, Kaaks R, Tjonneland A, Severi G, Perduca V, Boutron-Ruault MC, Weiderpass E, Ferrari P, Johnson T et al: Mitochondrial DNA Copy-Number Variation and Pancreatic Cancer Risk in the Prospective EPIC Cohort. Cancer Epidemiol Biomarkers Prev 2020, 29(3):681-686.

15. Xu J, Chang WS, Tsai CW, Bau DT, Davis JW, Thompson TC, Logothetis CJ, Gu J: Mitochondrial DNA copy number in peripheral blood leukocytes is associated with biochemical recurrence in prostate cancer patients in African Americans. Carcinogenesis 2020, 41(3):267-273.

16. Yang K, Li X, Forman MR, Monahan PO, Graham BH, Joshi A, Song M, Hang D, Ogino S, Giovannucci EL et al: Pre-diagnostic leukocyte mitochondrial DNA copy number and colorectal cancer risk. Carcinogenesis 2019, 40(12):1462-1468.

17. Xu E, Sun W, Gu J, Chow WH, Ajani JA, Wu X: Association of mitochondrial DNA copy number in peripheral blood leukocytes with risk of esophageal adenocarcinoma. Carcinogenesis 2013, 34(11):2521-2524.

18. Qu F, Chen Y, Wang X, He X, Ren T, Huang Q, Zhang J, Liu X, Guo X, Gu J et al: Leukocyte mitochondrial DNA content: a novel biomarker associated with prognosis and therapeutic outcome in colorectal cancer. Carcinogenesis 2015, 36(5):543-552.

Page $7 / 13$ 
19. He X, Qu F, Zhou F, Zhou X, Chen Y, Guo X, Li J, Huang Q, Yang Y, Lyu Z et al: High leukocyte mtDNA content contributes to poor prognosis through ROSmediated immunosuppression in hepatocellular carcinoma patients. Oncotarget 2016, 7(16):22834-22845.

20. Chen Y, Zhang J, Huang X, Zhang J, Zhou X, Hu J, Li G, He S, Xing J: High leukocyte mitochondrial DNA content contributes to poor prognosis in glioma patients through its immunosuppressive effect. Br J Cancer 2015, 113(1):99-106.

21. Xia P, An HX, Dang CX, Radpour R, Kohler C, Fokas E, Engenhart-Cabillic R, Holzgreve W, Zhong XY: Decreased mitochondrial DNA content in blood samples of patients with stage I breast cancer. BMC Cancer 2009, 9:454.

22. Edge SB, Compton CC: The American Joint Committee on Cancer: the 7th edition of the AJCC cancer staging manual and the future of TNM. Ann Surg Oncol 2010, 17(6):1471-1474.

23. Goldhirsch A, Winer EP, Coates AS, Gelber RD, Piccart-Gebhart M, Thurlimann B, Senn HJ, Panel m: Personalizing the treatment of women with early breast cancer: highlights of the St Gallen International Expert Consensus on the Primary Therapy of Early Breast Cancer 2013. Ann Onco/ 2013, 24(9):2206-2223.

24. Du R, Lu C, Jiang Z, Li S, Ma R, An H, Xu M, An Y, Xia Y, Jin L et al: Efficient typing of copy number variations in a segmental duplication-mediated rearrangement hotspot using multiplex competitive amplification. J Hum Genet 2012, 57(8):545-551.

25. Gourgou-Bourgade S, Cameron D, Poortmans P, Asselain B, Azria D, Cardoso F, A'Hern R, Bliss J, Bogaerts J, Bonnefoi H et al: Guidelines for time-to-event end point definitions in breast cancer trials: results of the DATECAN initiative (Definition for the Assessment of Time-to-event Endpoints in CANcer trials)dagger. Ann Oncol 2015, 26(5):873-879.

26. Lee HC, Yin PH, Lu CY, Chi CW, Wei YH: Increase of mitochondria and mitochondrial DNA in response to oxidative stress in human cells. Biochem J 2000, 348 Pt 2:425-432.

27. Shen M, Zhang L, Bonner MR, Liu CS, Li G, Vermeulen R, Dosemeci M, Yin S, Lan Q: Association between mitochondrial DNA copy number, blood cell counts, and occupational benzene exposure. Environ Mol Mutagen 2008, 49(6):453-457.

28. Liu CS, Tsai CS, Kuo CL, Chen HW, Lii CK, Ma YS, Wei YH: Oxidative stress-related alteration of the copy number of mitochondrial DNA in human leukocytes. Free Radic Res 2003, 37(12):1307-1317.

29. Jain M, Rivera S, Monclus EA, Synenki L, Zirk A, Eisenbart J, Feghali-Bostwick C, Mutlu GM, Budinger GR, Chandel NS: Mitochondrial reactive oxygen species regulate transforming growth factor-beta signaling. J Biol Chem 2013, 288(2):770-777.

30. Giannelli G, Villa E, Lahn M: Transforming growth factor-beta as a therapeutic target in hepatocellular carcinoma. Cancer Res 2014, 74(7):1890-1894.

31. Weerts MJ, Sieuwerts AM, Smid M, Look MP, Foekens JA, Sleijfer S, Martens JW: Mitochondrial DNA content in breast cancer. Impact on in vitro and in vivo phenotype and patient prognosis. Oncotarget 2016, 7(20):29166-29176.

32. Bai RK, Chang J, Yeh KT, Lou MA, Lu JF, Tan DJ, Liu H, Wong LJ: Mitochondrial DNA content varies with pathological characteristics of breast cancer. J Oncol 2011, 2011:496189.

33. Rai NK, Panjwani G, Ghosh AK, Haque R, Sharma LK: Analysis of mitochondrial DNA copy number variation in blood and tissue samples of metastatic breast cancer patients (A pilot study). Biochem Biophys Rep 2021, 26:100931.

34. Chen N, Wen S, Sun X, Fang Q, Huang L, Liu S, Li W, Qiu M: Elevated Mitochondrial DNA Copy Number in Peripheral Blood and Tissue Predict the Opposite Outcome of Cancer. A Meta-Analysis. Sci Rep 2016, 6:37404.

35. D'Erchia AM, Atlante A, Gadaleta G, Pavesi G, Chiara M, De Virgilio C, Manzari C, Mastropasqua F, Prazzoli GM, Picardi E et al: Tissue-specific mtDNA abundance from exome data and its correlation with mitochondrial transcription, mass and respiratory activity. Mitochondrion 2015, 20:13-21.

36. Fernandez-Vizarra E, Enriquez JA, Perez-Martos A, Montoya J, Fernandez-Silva P: Tissue-specific differences in mitochondrial activity and biogenesis. Mitochondrion 2011, 11(1):207-213.

37. Pedram A, Razandi M, Wallace DC, Levin ER: Functional estrogen receptors in the mitochondria of breast cancer cells. Mol Biol Cell 2006, 17(5):21252137.

38. Behera MA, Dai Q, Garde R, Saner C, Jungheim E, Price TM: Progesterone stimulates mitochondrial activity with subsequent inhibition of apoptosis in MCF-10A benign breast epithelial cells. Am J Physiol Endocrinol Metab 2009, 297(5):E1089-1096.

39. Gao W, Wu MH, Wang N, Ying MZ, Zhang YY, Hua J, Chuan L, Wang YJ: Mitochondrial transcription factor A contributes to cisplatin resistance in patients with estrogen receptorpositive breast cancer. Mol Med Rep 2016, 14(6):5304-5310.

40. Cam H, Balciunaite E, Blais A, Spektor A, Scarpulla RC, Young R, Kluger Y, Dynlacht BD: A common set of gene regulatory networks links metabolism and growth inhibition. Mol Cell 2004, 16(3):399-411.

41. Ishikawa K, Takenaga K, Akimoto M, Koshikawa N, Yamaguchi A, Imanishi H, Nakada K, Honma Y, Hayashi J: RoS-generating mitochondrial DNA mutations can regulate tumor cell metastasis. Science 2008, 320(5876):661-664.

\section{Tables}

Table 1 Patients' clinicopathological characteristics and clinical outcome 


\begin{tabular}{|c|c|c|c|c|c|c|c|c|c|}
\hline Variables & Patients & 5-Year iDFS & & 5-Year DDFS & & 5-Year BCSS & & 5-Year OS & \\
\hline & $N=661$ & Events & LogRank $P$ & Events & LogRank $P$ & Events & LogRank $P$ & Events & LogRank $P$ \\
\hline $\mathrm{Ag}$ at diagnosis & & & 0.136 & & 0.331 & & 0.754 & & 0.934 \\
\hline$\leq 40$ & 207 & 97 & & 86 & & 56 & & 56 & \\
\hline$\otimes 40$ & 454 & 182 & & 168 & & 120 & & 124 & \\
\hline Tumor size $₫ \mathrm{~cm} \rrbracket$ & & & $\varangle 0.001$ & & $\llbracket 0.001$ & & $₫ 0.001$ & & $₫ 0.001$ \\
\hline$\leq 2$ & 211 & 55 & & 48 & & 34 & & 34 & \\
\hline$\otimes 2$ & 450 & 224 & & 206 & & 142 & & 146 & \\
\hline Nodal status & & & $\otimes 0.001$ & & $\llbracket 0.001$ & & $\llbracket 0.001$ & & $\otimes 0.001$ \\
\hline Negative & 255 & 66 & & 55 & & 30 & & 33 & \\
\hline Positive & 406 & 213 & & 199 & & 146 & & 147 & \\
\hline Clinical stage & & & $\triangle 0.001$ & & $\llbracket 0.001$ & & $\llbracket 0.001$ & & $\otimes 0.001$ \\
\hline I & 127 & 21 & & 17 & & 12 & & 12 & \\
\hline$\|+\mid\|$ & 534 & 258 & & 237 & & 164 & & 168 & \\
\hline Grade & & & $₫ 0.001$ & & $\otimes 0.001$ & & $\otimes 0.001$ & & $\varangle 0.001$ \\
\hline$|+| \mid$ & 500 & 190 & & 172 & & 118 & & 121 & \\
\hline III & 161 & 89 & & 82 & & 58 & & 59 & \\
\hline $\mathrm{HR}$ & & & $\varangle 0.001$ & & $\bowtie 0.001$ & & $\llbracket 0.001$ & & $\llbracket 0.001$ \\
\hline Negative & 235 & 129 & & 121 & & 99 & & 100 & \\
\hline Positive & 426 & 150 & & 133 & & 77 & & 80 & \\
\hline HER2 & & & $\otimes 0.001$ & & $\llbracket 0.001$ & & $\llbracket 0.001$ & & $\otimes 0.001$ \\
\hline Negative & 467 & 170 & & 152 & & 103 & & 106 & \\
\hline Positive & 194 & 109 & & 102 & & 73 & & 74 & \\
\hline Subtype & & & $₫ 0.001$ & & $\otimes 0.001$ & & $\otimes 0.001$ & & $\varangle 0.001$ \\
\hline Luminal A & 123 & 18 & & 17 & & 8 & & 8 & \\
\hline Luminal B & 303 & 132 & & 116 & & 69 & & 72 & \\
\hline HER2+ & 101 & 64 & & 59 & & 48 & & 48 & \\
\hline Triple negative & 134 & 65 & & 62 & & 51 & & 52 & \\
\hline
\end{tabular}

Abbreviations: iDFS, invasive disease-free survival; DDFS, distant disease-free survival; BCSS, breast cancer special survival; OS, overall survival; HR, hazard ratio; HER2: human epidermal growth factor-2.

Table 2 Associations between mtDNA copy number and clinicopathological characteristics in BC patients. 


\begin{tabular}{|c|c|c|c|}
\hline Characteristics & 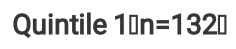 & Quintile 2-5 $\square n=529 \square$ & $P$-value \\
\hline Age at diagnosis & & & 0.753 \\
\hline$\leq 40$ & $43(32.6 \%)$ & $164(31.0 \%)$ & \\
\hline$\otimes 40$ & $89(67.4 \%)$ & $365(69.0 \%)$ & \\
\hline Tumor size $\llbracket \mathrm{cm} \rrbracket$ & & & 0.348 \\
\hline$\leq 2$ & $47(35.6 \%)$ & $164(31.0 \%)$ & \\
\hline$凶 2$ & 85 (64.4\%) & $365(69.0 \%)$ & \\
\hline Nodal status & & & 0.272 \\
\hline Negative & $45(34.1 \%)$ & $210(39.7 \%)$ & \\
\hline Positive & $87(65.9 \%)$ & $319(60.3 \%)$ & \\
\hline Clinical stage & & & 0.622 \\
\hline I & $23(17.4 \%)$ & 104 (19.7\%) & \\
\hline$\|+\|$ & $109(82.6 \%)$ & 425 (80.3\%) & \\
\hline Grade & & & 0.041 \\
\hline $1+\|$ & $109(82.6 \%)$ & 391 (73.9\%) & \\
\hline III & $23(17.4 \%)$ & $138(26.1 \%)$ & \\
\hline HR & & & 0.186 \\
\hline Negative & $40(30.3 \%)$ & $195(36.9 \%)$ & \\
\hline Positive & $92(69.7 \%)$ & $334(63.1 \%)$ & \\
\hline HER2 & & & 0.749 \\
\hline Negative & $95(72.0 \%)$ & 372 (70.3\%) & \\
\hline Positive & $37(28.0 \%)$ & $157(29.7 \%)$ & \\
\hline Subtype & & & 0.393 \\
\hline Luminal A & $28(21.2 \%)$ & $95(18.0 \%)$ & \\
\hline Luminal B & $64(48.4 \%)$ & $239(45.2 \%)$ & \\
\hline HER2+ & $20(15.2 \%)$ & $81(15.3 \%)$ & \\
\hline Triple-negative & $20(15.2 \%)$ & $114(21.5 \%)$ & \\
\hline
\end{tabular}

Abbreviations: mtDNA, mitochondrial DNA; HR, hazard ratio; HER2: human epidermal growth factor-2.

Table 3 Results of cox model on breast cancer risk for the quintile 2 to 5 of mtDNA copy number versus quintile 1 (as reference category)

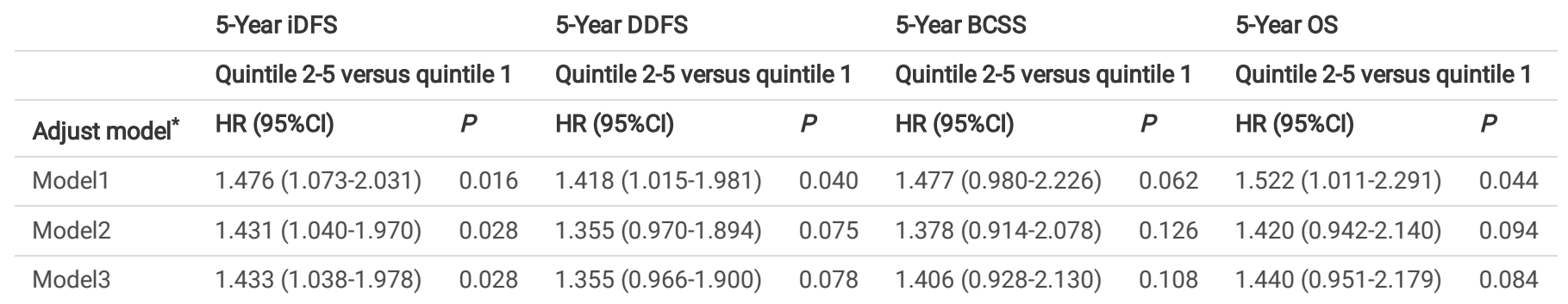

Abbreviations: iDFS, invasive disease-free survival; DDFS, distant disease-free survival; BCSS, breast cancer special survival; OS, overall survival; Cl, confidence interval; mtDNA, mitochondrial DNA; HR, hazard ratio.

* Model 1: adjusted for age at diagnosis; Model 2: same as model 1, plus hormone receptor status. HER2 status; Model 3: same as model 2, plus tumor size, Lymph node involvement, grade. 
Table 4 Hazard ratio of tumor survival for all 661 patients with breast cancer according to the mtDNA copy number in different subgroups stratified by clinical parameters

\begin{tabular}{|c|c|c|c|c|c|c|c|c|c|c|c|c|}
\hline Variables & & 5-Year iDFS & & & $\begin{array}{l}\text { 5-Year } \\
\text { DDFS }\end{array}$ & & & 5-Year BCSS & & & 5-Year OS & \\
\hline & & $\begin{array}{l}\text { Ajusted } \\
\mathrm{HR}^{*} \mathrm{\square} 95 \% \mathrm{Cl} \square\end{array}$ & $P$ & $\begin{array}{l}P \text { for } \\
\text { interaction }\end{array}$ & $\begin{array}{l}\text { Ajusted } \\
\text { HR* }^{*} 95 \% \mathrm{Cl}[\end{array}$ & $P$ & $\begin{array}{l}P \text { for } \\
\text { interaction }\end{array}$ & $\begin{array}{l}\text { Ajusted HR* } \\
\text { प95\%Cl口] }\end{array}$ & $P$ & $\begin{array}{l}P \text { for } \\
\text { interaction }\end{array}$ & $\begin{array}{l}\text { Ajusted } \\
\left.\mathrm{HR}^{*} \mathrm{\square} 95 \% \mathrm{Cl}\right]\end{array}$ & $P$ \\
\hline Age & & & & 0.266 & & & 0.275 & & & 0.204 & & \\
\hline$\leq 40$ & 207 & $\begin{array}{l}1.847 \\
(1.047- \\
3.259)\end{array}$ & 0.034 & & $\begin{array}{l}1.810 \\
(0.982- \\
3.336)\end{array}$ & 0.057 & & $\begin{array}{l}2.096 \\
(0.946- \\
4.645)\end{array}$ & 0.068 & & $\begin{array}{l}2.096 \\
(0.946- \\
4.645)\end{array}$ & 0.068 \\
\hline$\otimes 40$ & 454 & $\begin{array}{l}1.262 \\
(0.853- \\
1.869)\end{array}$ & 0.244 & & $\begin{array}{l}1.178 \\
(0.784- \\
1.769)\end{array}$ & 0.430 & & $\begin{array}{l}1.204 \\
(0.738- \\
1.964)\end{array}$ & 0.457 & & $\begin{array}{l}1.248 \\
(0.767- \\
2.031)\end{array}$ & 0.373 \\
\hline HR & & & & 0.687 & & & 0.334 & & & 0.028 & & \\
\hline Negative & 235 & $\begin{array}{l}1.390 \\
(0.847- \\
2.282)\end{array}$ & 0.193 & & $\begin{array}{l}1.177 \\
(0.714- \\
1.939)\end{array}$ & 0.522 & & $\begin{array}{l}0.988 \\
(0.586- \\
1.667)\end{array}$ & 0.964 & & $\begin{array}{l}0.988 \\
(0.586- \\
1.665)\end{array}$ & 0.963 \\
\hline Positive & 426 & $\begin{array}{l}1.473 \\
(0.962- \\
2.256)\end{array}$ & 0.075 & & $\begin{array}{l}1.520 \\
(0.958- \\
2.411)\end{array}$ & 0.075 & & $\begin{array}{l}2.340 \\
(1.163- \\
4.708)\end{array}$ & 0.017 & & $\begin{array}{l}2.446 \\
(1.218- \\
4.913)\end{array}$ & 0.011 \\
\hline HER2 & & & & 0.501 & & & 0.232 & & & 0.640 & & \\
\hline Negative & 467 & $\begin{array}{l}1.551 \\
(1.018- \\
2.362)\end{array}$ & 0.041 & & $\begin{array}{l}1.573 \\
(0.997- \\
2.482)\end{array}$ & 0.051 & & $\begin{array}{l}1.497 \\
(0.860- \\
2.606)\end{array}$ & 0.153 & & $\begin{array}{l}1.535 \\
(0.884- \\
2.667)\end{array}$ & 0.128 \\
\hline Positive & 194 & $\begin{array}{l}1.336 \\
(0.808- \\
2.208)\end{array}$ & 0.258 & & $\begin{array}{l}1.135 \\
(0.684- \\
1.884)\end{array}$ & 0.623 & & $\begin{array}{l}1.287 \\
(0.684- \\
2.420)\end{array}$ & 0.434 & & $\begin{array}{l}1.318 \\
(0.702- \\
2.475)\end{array}$ & 0.390 \\
\hline Grade & & & & 0.795 & & & 0.828 & & & 0.709 & & \\
\hline $1+\|$ & 500 & $\begin{array}{l}1.374 \\
(0.954- \\
1.981)\end{array}$ & 0.088 & & $\begin{array}{l}1.355 \\
(0.920- \\
1.994)\end{array}$ & 0.124 & & $\begin{array}{l}1.427 \\
(0.887- \\
2.295)\end{array}$ & 0.142 & & $\begin{array}{l}1.467 \\
(0.914- \\
2.356)\end{array}$ & 0.112 \\
\hline III & 161 & $\begin{array}{l}1.596 \\
(0.793- \\
3.210)\end{array}$ & 0.190 & & $\begin{array}{l}1.290 \\
(0.638- \\
2.610)\end{array}$ & 0.478 & & $\begin{array}{l}1.347 \\
(0.567- \\
3.200)\end{array}$ & 0.499 & & $\begin{array}{l}1.375 \\
(0.580- \\
3.262)\end{array}$ & 0.469 \\
\hline $\begin{array}{l}\text { Tumor } \\
\text { sizes }\end{array}$ & & & & 0.347 & & & 0.329 & & & 0.942 & & \\
\hline$\leq 2$ & 211 & $\begin{array}{l}2.053 \\
(0.997- \\
4.230)\end{array}$ & 0.051 & & $\begin{array}{l}2.042 \\
(0.946- \\
4.405)\end{array}$ & 0.068 & & $\begin{array}{l}1.519 \\
(0.657- \\
3.508)\end{array}$ & 0.328 & & $\begin{array}{l}1.519 \\
(0.657- \\
3.508)\end{array}$ & 0.328 \\
\hline$\otimes 2$ & 450 & $\begin{array}{l}1.302 \\
(0.907- \\
1.868)\end{array}$ & 0.152 & & $\begin{array}{l}1.229 \\
(0.843- \\
1.793)\end{array}$ & 0.283 & & $\begin{array}{l}1.430 \\
(0.885- \\
2.312)\end{array}$ & 0.144 & & $\begin{array}{l}1.479 \\
(0.916- \\
2.387)\end{array}$ & 0.109 \\
\hline $\begin{array}{l}\text { Nodal } \\
\text { status }\end{array}$ & & & & 0.170 & & & 0.240 & & & 0.208 & & \\
\hline Negative & 255 & $\begin{array}{l}0.941 \\
(0.502- \\
1.767)\end{array}$ & 0.851 & & $\begin{array}{l}0.882 \\
(0.442- \\
1.759)\end{array}$ & 0.721 & & $\begin{array}{l}0.766 \\
(0.310- \\
1.892)\end{array}$ & 0.564 & & $\begin{array}{l}0.864 \\
(0.354- \\
2.107)\end{array}$ & 0.747 \\
\hline Positive & 406 & $\begin{array}{l}1.603 \\
(1.101- \\
2.334)\end{array}$ & 0.013 & & $\begin{array}{l}1.499 \\
(1.016- \\
2.211)\end{array}$ & 0.041 & & $\begin{array}{l}1.577 \\
(0.986- \\
2.522)\end{array}$ & 0.057 & & $\begin{array}{l}1.597 \\
(0.999- \\
2.553)\end{array}$ & 0.050 \\
\hline
\end{tabular}

Abbreviations: iDFS, invasive disease-free survival; DDFS, distant disease-free survival; BCSS, breast cancer special survival; OS, overall survival; Cl, confidence interval; mtDNA, mitochondrial DNA; HR, hazard ratio.

* adjusted for age at diagnosis, tumor size, Lymph node involvement, grade, hormone receptor status, and HER2 status, except for the interaction factor. 
Table 5 Results of stepwise cox regression analysis on breast cancer-specific survival and overall survival in HR-positive breast cancer patients.

\begin{tabular}{|c|c|c|}
\hline Factor (in order of entry) & $\mathrm{HR}(95 \% \mathrm{Cl})$ & $P$ (in final modal) \\
\hline \multicolumn{3}{|l|}{ 5-Year Breast Cancer-Specific Survival } \\
\hline 1. Nodal status $₫$ positive vs negative $\mathbb{}$ & $4.148(2.186-7.783)$ & $\varangle 0.001$ \\
\hline 2. Grade $\| I I I$ vs I+IIU & $1.859(1.138-3.035)$ & 0.013 \\
\hline  & $2.420(1.204-4.863)$ & 0.013 \\
\hline
\end{tabular}

\section{5-Year Overall Survival}

\begin{tabular}{|c|c|c|}
\hline 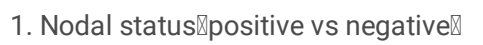 & $3.454(1.900-6.279)$ & $\varangle 0.001$ \\
\hline 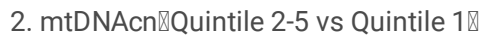 & $2.464(1.227-4.948)$ & 0.011 \\
\hline 3. Grade $\mathbb{I I I}$ vs I+I\| & $1.705(1.046-2.777)$ & 0.032 \\
\hline 4. HER2\positive vs negative》 & $1.537(0.959-2.463)$ & 0.073 \\
\hline
\end{tabular}

\section{Figures}


Figure 1

Kaplan-Meier curves for iDFS (a), DDFS (b), BCSS (c), and OS (d) due to breast cancer by Quintile 2-5 AND Quintile 1 of mitochondrial DNA copy number. Abbreviations: iDFS, invasive disease-free survival; DDFS, distant disease-free survival; BCSS, breast cancer special survival; OS, overall survival. 


\section{Supplementary Files}

This is a list of supplementary files associated with this preprint. Click to download.

- Tables1.docx

- Tables2.docx

- Figures1.docx 